\title{
Selective tissue targeting of synthetic nucleic acid drugs
}

\author{
Punit P. Seth, Michael Tanowitz, and C. Frank Bennett
}

Ionis Pharmaceuticals Inc., Carlsbad, California, USA.

\begin{abstract}
Antisense oligonucleotides (ASOs) are chemically synthesized nucleic acid analogs designed to bind to RNA by Watson-Crick base pairing. Following binding to the targeted RNA, the ASO perturbs RNA function by promoting selective degradation of the targeted RNA, altering RNA intermediary metabolism, or disrupting function of the RNA. Most antisense drugs are chemically modified to enhance their pharmacological properties and for passive targeting of the tissues of therapeutic interest. Recent advances in selective tissue targeting have resulted in a newer generation of ASO drugs that are more potent and better tolerated than previous generations, spawning renewed interest in identifying selective ligands that enhance targeted delivery of ASOs to tissues.
\end{abstract}

\section{Introduction to tissue targeting}

Selective tissue and cellular targeting of therapeutic agents has been an active area of investigation for decades. Paul Ehrlich first coined the term "magic bullet" - a therapeutic agent that might be selectively designed to target an infectious agent without affecting the host. This concept has been extended to include a number of therapeutic strategies, with cancer perhaps receiving the most attention and, as a result, the most progress. The reasons for pursuing targeted therapeutics include (a) to enhance therapeutic potency by directing more of the administered dose to the target tissue, (b) to enhance delivery to the appropriate subcellular compartment, (c) to reduce toxicity by decreasing drug concentrations in nontargeted tissue, (d) to enhance the agent's pharmacokinetic profile by increasing circulation time, (e) to broaden the agent's therapeutic application by increasing the repertoire of cells or tissues targeted, and (f) to decrease cost by lowering the total dose administered.

Both physical and chemical methods can achieve specific tissue targeting. Aerosol delivery to the lung and intrathecal administration to cerebrospinal fluid are examples of methods of physical delivery to specific tissues. Chemical tissue targeting can occur either passively or actively. Examples of passive targeting include enhancement of the therapeutic agent's binding to plasma proteins to increase its circulation time; formulation in lipid or other types of nanoparticles; and direct conjugation to plasma proteins or PEG, which increases the agent's molecular size and decreases clearance by glomerular filtration and the reticuloendothelial system (RES). Active targeting can be achieved by attachment of

Conflict of interest: All authors are employees of lonis Pharmaceuticals, own stock in the company, and are compensated by lonis. Authors are not compensated by another organization. CFB was recently a recipient of the Breakthrough Award. All work is supported by lonis Pharmaceuticals. Authors do not receive research support from another organization. All the authors are listed as co-inventors on multiple issued US and international patents and patent applications (W02017192820A1, W02014179620A1, and US7399845B2 to PPS, MT, and (FB). All authors are also inventors on additional patent applications not listed here, as those topics are not discussed in this Review.

Reference information: J Clin Invest. 2019;129(3):915-925.

https://doi.org/10.1172/JCI125228. the drug to a ligand that binds to and is internalized by a specific receptor expressed on the targeted cell. Perhaps the most developed example of active targeting is the use of antibodies to deliver chemotherapeutic agents to cancer cells. Currently, four approved drugs use a cytotoxic agent covalently attached to an antibody as the targeting moiety, and more than 50 additional antibody-drug combinations are in development $(1,2)$.

This Review focuses on various strategies to target synthetic oligonucleotides to tissues and cell types to achieve antisense effects. Antisense oligonucleotides (ASOs) are designed to bind to nuclear or cytoplasmic RNA and so must be internalized by cells and escape at least one cellular membrane to access their complementary RNA. As we discuss, this creates unique challenges for tissue targeting in that hydrophilic ASOs must bind to acceptors expressed on the cell surface and also be transported into the cell and escape through a hydrophobic membrane bilayer to gain access to their cognate RNA receptor.

\section{ASO therapeutics}

A comprehensive review of antisense technology is beyond the scope of this Review, and the reader is referred to several recent reviews for background $(3,4)$. Briefly, ASOs are chemically synthesized oligonucleotides that bind RNA through Watson-Crick base pairing, which provides their specificity. Once bound to the targeted RNA, the oligonucleotide can either modulate RNA function through nondegradative mechanisms or promote RNA degradation through endogenous enzymes (5). Examples of nondegradative mechanisms include blocking of translation, enhancement of translation, modulation of RNA splicing, and blocking of microRNA functions. In each case the oligonucleotide binds to the targeted RNA in a sequence-specific manner and disrupts either RNA-protein binding or RNA structure. The most clinically advanced nondegradative mechanism is modulation of RNA splicing, with two approved drugs on the market: eteplirsen for Duchenne muscular dystrophy and nusinersen for the treatment of spinal muscular atrophy $(6,7)$. RNA degradative mechanisms rely on endogenous RNA nucleases to specifically degrade the targeted RNA at the site of ASO hybridization. 
Table 1. Chemical modifications commonly used in nucleic acid therapeutics

\begin{tabular}{|c|c|c|c|c|c|}
\hline Modification & Abbreviation & Position & Nuclease stability & Protein binding & RNA binding ( $\left.{ }^{\circ} \mathrm{C} / \mathrm{mod}.\right)$ \\
\hline 2'-0-Methyl RNA & $\mathrm{OMe}$ & Sugar & + & $+/-$ & +1 \\
\hline 2'-Fluoro RNA & $F$ & Sugar & $+/-$ & + & +1 \\
\hline 2'-0-Methoxyethyl RNA & MOE & Sugar & ++ & $+1-$ & +1 to 1.5 \\
\hline Locked nucleic acid & LNA & Sugar & ++ & $+1-$ & +3 to 5 \\
\hline Constrained ethyl BNA & cEt & Sugar & +++ & $+1-$ & +3 to 5 \\
\hline Morpholino & PMO & Sugar and backbone & ++++ & - & +1 \\
\hline Phosphorothioate & PS & Backbone & ++ & +++ & -0.5 \\
\hline Cholesterol, lipids & NA & Conjugate & ++ & +++ & NA \\
\hline
\end{tabular}

BNA, 2',4'-bridged nucleic acid; PMO, phosphorodiamidate morpholino.

Two cellular nucleases are broadly used to promote selective degradation of the targeted RNA, RNase H1 and Ago2. RNase H1 recognizes the RNA-ASO heteroduplex, degrading the RNA and leaving the ASO intact to bind to another targeted RNA. Approaches using Ago2, the protein effector of the RNA interference pathway, administer an RNA-RNA duplex complex (siRNA) to cells or an organism. Once inside the cytoplasm, the duplex is unwound, with one strand (the antisense or guide strand) interacting with the nuclease Ago2 to form the RNA-induced silencing complex (RISC) (8).

To date, six antisense drugs have been approved by various regulatory agencies (with an additional drug under review) to treat diseases spanning viral infections, hyperlipidemias, and neurological diseases (3). Well over 50 additional ASO drugs are in various stages of clinical trials, including several in pivotal studies (summarized in ref. 3). All approved antisense drugs and drugs under regulatory review rely on passive oligonucleotide delivery to the target tissues, which include liver, skeletal muscle, neurons, and retinal cells. Recent clinical results demonstrate the utility of tissue targeting for ASOs, demonstrating greatly enhanced potency compared with nontargeted ASOs $(9,10)$. These results have incentivized researchers to identify additional safe and effective targeting ligands for ASOs.

\section{Introduction to chemical modifications of oligonucleotides and formulations}

Therapeutic oligonucleotides are used as single- or doublestranded designs, the choice of which is dictated by the antisense mechanism being harnessed. Unmodified single- or double-stranded oligonucleotides typically do not possess sufficient drug-like attributes to be used as effective therapeutics (11). They lack sufficient metabolic stability in extracellular or intracellular fluids or suitable pharmacokinetic properties. Some nucleic acids can also trigger biological sensors like TLRs and RIG-I, producing undesired immune modulatory effects $(12,13)$. To address these limitations, medicinal chemists devised chemical modification or formulation strategies to help deliver these agents for therapeutic applications (Figure 1).

Modifications that enhance nuclease stability and RNA affinity. DNA and RNA represent the starting point for introducing chemical modifications into nucleic acids (Table 1). Modifications can be made to the backbone, sugar, or nucleobases of
DNA and RNA (11). Typically, modifications at the 2 ' position of the furanose ring in natural nucleic acids enhance affinity for complementary RNA by shifting the furanose conformation into the $\mathrm{C}^{\prime}$-endo sugar pucker. Examples include 2'-O-methyl (OMe), 2'-fluoro (F), and 2'-O-methoxyethyl (MOE) RNA. Conformational restriction of the furanose ring into the $\mathrm{C} 3^{\prime}$-endo sugar pucker gives modifications such as locked nucleic acid (LNA) $(14,15)$ and constrained ethyl (cEt) (16), which provide further increases in binding affinity for complementary RNA. All these modifications typically enhance metabolic stability, as the steric bulk of the modification hinders attack by nucleases, which hydrolyze the internucleosidic phosphodiester linkages (17). Phosphorodiamidate morpholinos (PMOs) represent another modification that enhances metabolic stability and affinity for RNA by replacing the sugar and backbone with a morpholino ring system (18).

Modifications to enhance nuclease stability and protein affinity. While each modification discussed above enhances metabolic stability and RNA-binding affinity of nucleic acids, the ability to enhance interactions with proteins is a distinct property of the phosphorothioate (PS) backbone modification (19). In the PS linkage, one of the nonbridging oxygen atoms of the natural phosphodiester linkage is replaced with a sulfur atom. PS-modified single-stranded oligonucleotides show enhanced avidity for a host of plasma, cell surface, and intracellular proteins (4, 20). While the molecular origins of the enhanced protein-binding properties of PS oligonucleotides are not well understood, they likely result from the anionic/hydrophobic nature of the sulfur atom in the PS modification (21). At least 12-15 PS linkages are required for effective protein binding, suggesting an avidity effect wherein each PS linkage contributes a small percentage to the overall interaction (22). Attaching hydrophobic moieties such as fatty acids, cholesterol, or tocopherol at either terminus of single- or double-stranded oligonucleotides represents another strategy to enhance the protein-binding properties of nucleic acid drugs (23).

Nanoparticulate formulations. Nucleic acids with no or limited chemical modifications can be encapsulated within nanoparticulate formulations to aid their delivery (Figure 1). Encapsulating the oligonucleotide protects it from nuclease-mediated degradation in the serum and in endosomal compartments. The lipid nanoparticle (LNP) platform represents the leading formulation 
A

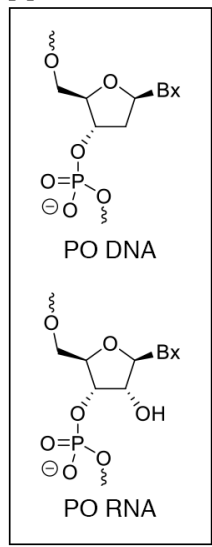

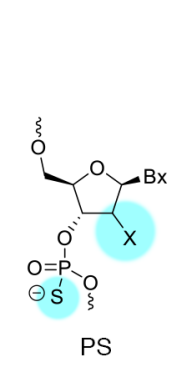

Enhance nuclease stability and affinity for proteins

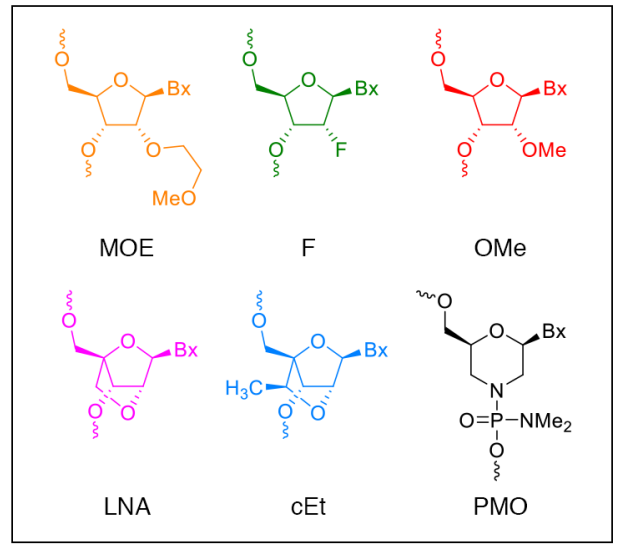

Enhance nuclease stability and affinity for RNA
B

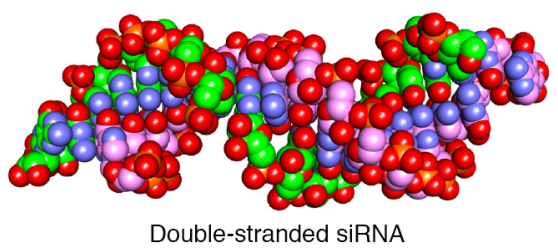

C

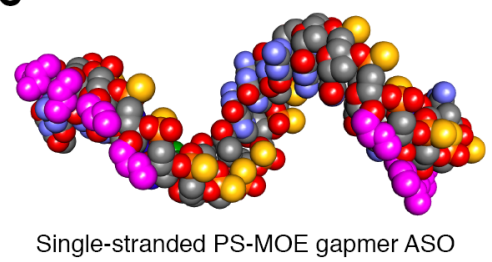

D

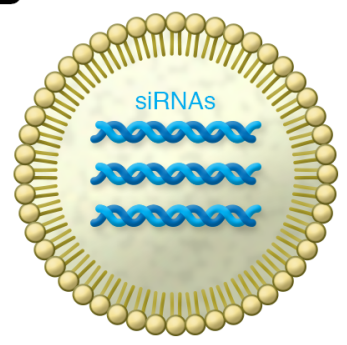

LNP
E

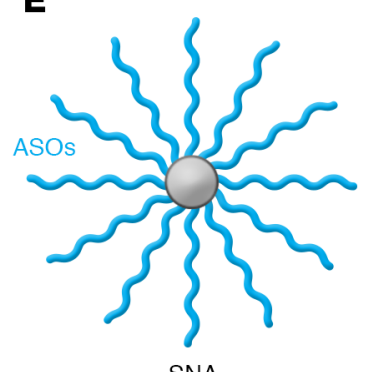

platform for delivery of nucleic acid drugs (24). LNPs typically comprise the nucleic acid drug, an ionizable amino lipid, distearoylphosphatidylcholine, cholesterol, and a PEG lipid, which form approximately 100-nm-diameter nanoparticles. Spherical nucleic acids represent another distinct class of nanoparticulate formulation with a hydrophobic core and a hydrophilic nucleic acid corona. Nanoparticulate formulations are injected intravenously, and their distribution is limited to phagocytic macrophages or tissues with sinusoidal capillary architectures like the liver. In contrast, PS-modified ASOs can be injected subcutaneously and distribute broadly to almost all tissues in an animal (25).

\section{Tissue and cellular barriers to activity}

To achieve efficacy, an ASO has to travel from its site of injection to its RNA target inside cells within tissues. In the process, the ASO must avoid digestion by blood- and tissue-borne nucleases, scavenging by the RES (unless the gene target resides in these cells), and excretion into urine. Next, the drug must traverse the capillary endothelium, leaving the blood compartment, and enter the tissue interstitium. Upon arrival at the cell surface of interest, the ASO adheres to protein "acceptors" on the cell surface that
Figure 1. Chemical modification and formulation strategies for delivery of oligonucleotide drugs. (A) Chemical modifications commonly used to impart drug-like properties to oligonucleotide drugs. (B) Double-stranded RNA (pink and green carbons illustrate individual strands). (C) Single-stranded PS-modified MOE gapmer ASO (yellow spheres show PS, and pink spheres show MOE modifications). ( $\mathbf{D}$ and $\mathbf{E})$ LNP-encapsulated double-stranded oligonucleotides (D) versus single-stranded oligonucleotide-coated SNAs (E). cEt constrained ethyl; F, 2'-fluoro; LNA, locked nucleic acid; LNP, lipid nanoparticle; MOE, 2'-O-methoxyethyl; OMe, 2'-O-methyl; PMO, phosphorodiamidate morpholino; PS, phosphorothioate; SNA, spherical nucleic acid.

facilitate cellular entry. Even after internalization, the nucleic acid drug must survive the aggressive nuclease milieu in endo-lysosomal compartments, escape the vesicular structures, gain entry into the cytosol/nucleus, and find the targeted RNA. Given these hurdles, it is amazing that antisense technology works, but overwhelming evidence supports this conclusion. In the following sections, we will discuss some of the tissue and cellular barriers that must be overcome to facilitate effective delivery, identifying strategies that may make these processes more efficient.

Kidney filtration. Unmodified nucleic acids are rapidly filtered and excreted in the urine (26). Chemically modified nucleic acids such as PS ASOs and lipid-modified siRNA, which associate with plasma proteins, demonstrate limited filtration and are efficiently reabsorbed by cells of the proximal tubules in the kidney (25). For nucleic acids encapsulated in nanoparticles, kidney filtration is not an issue, but distribution is typically limited to the liver and other cells of the RES.

Clearance by the RES. In the liver, the RES includes both liver sinusoidal endothelial cells (LSECs) and Kupffer cells (27). LSECs clear a variety of soluble waste macromolecules, including hyaluronic acid, glycosaminoglycans of the extracellular matrix, advanced glycation end products, oxidized LDL, and heparin (28). In contrast, Kupffer cells clear large insoluble waste fragments by phagocytosis. Other organs, like the spleen, lymph nodes (LNs), and BM, also express a mosaic of scavenger receptors that clear waste macromolecules and particulate products from blood (29). Reducing the surface charge on nanoparticle formulations and PEGylation can reduce RES clearance by mitigating interactions with scavenger receptors $(30,31)$.

Passage across the endothelium. If the nucleic acid drug survives kidney filtration and RES-mediated clearance, the capillary endothelium presents the next barrier to accessing the tissue interstitium (32). There are at least three distinct classes of capillary endothelium (33): the continuous endothelium in tissues such as the heart and skeletal muscle; fenestrated endothelium in tissues 
A
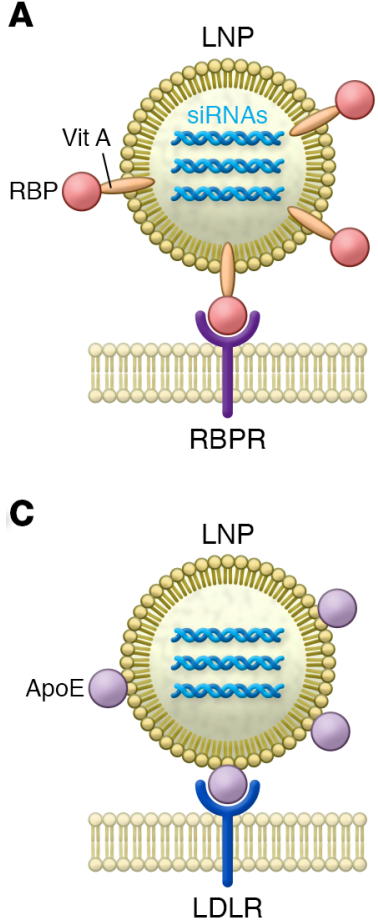

E

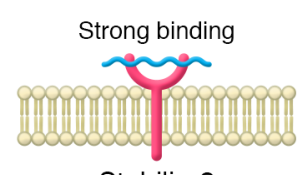

Stabilin-2

G

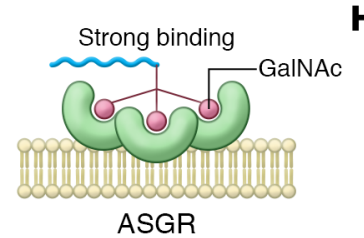

B

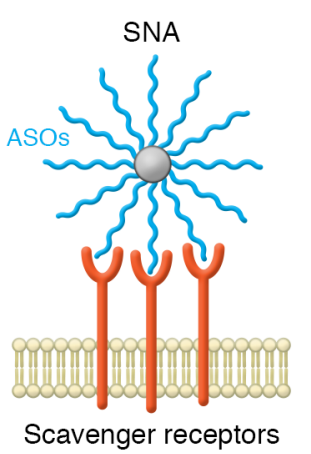

D

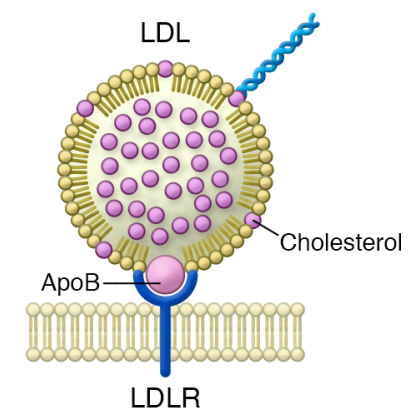

$\mathbf{F}$

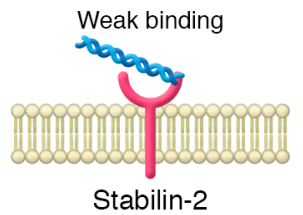

H

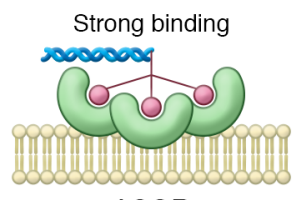

ASGR

such as the kidney, endocrine organs, and choroid plexus; and the sinusoidal endothelium in liver, lymphoid, and hematopoietic tissues. The nucleic acid drug can traverse this barrier in either a paracellular or a transcellular manner.

For chemically modified nucleic acid drugs (molecular weight 5-15 kDa) and nucleic acids formulated in nanoparticles $(\sim 100$ $\mathrm{nm}$ diameter), the paracellular route is essentially limited to tissues such as the liver where gaps between endothelial cells and an incomplete basement membrane facilitate the drug's passage from the bloodstream into the space of Disse (34). Presumably, the porous nature of the liver sinusoids provides direct access for macromolecular therapeutics to the hepatocyte cell surface. Sinusoids are also found in lymphoid tissues and in hematopoietic organs such as the spleen and the BM. Interestingly, all of these tissues represent sites of significant accumulation for some classes of nucleic acid therapeutics (25).

The fenestrated endothelium permits passage of molecules with molecular weights of approximately $5000 \mathrm{Da}$ or smaller through openings in the endothelial cells, but these molecules still need to traverse the intact basement membrane to access the tis-

Figure 2. Models for interactions of formulated, chemically modified, or ligand-conjugated oligonucleotides with cell surface receptors. (A) Vitamin A-LNPs interact with retinol-binding proteins (RBPs) in plasma and promote cellular uptake via RBP receptors (RBPRs). (B) SNAs interact with scavenger receptors via multivalent interactions to promote cellular uptake. (C and D) LDLR-mediated uptake of apolipoprotein E-decorated LNPs (C) or cholesterol-conjugated siRNA (D). (E and $\mathbf{F}$ ) Single-stranded PS ASOs (E) interact with stabilin receptors more efficiently than double-stranded oligonucleotides (F). (G and $\mathbf{H})$ Trivalent GalNAc-modified ASOs (C) and siRNA (H) interact with the ASGR with equal efficiency.

sue interstitium. Short nucleic acids can presumably exit through these openings, as evidenced by accumulation of PS ASOs in the kidneys. PS ASOs are filtered through the fenestrated endothelium in the glomerulus and reabsorbed in the proximal tubules (25). Nanoparticle formulations typically have reduced exposure to the kidney, as their size prevents glomerular filtration.

Tight junctions between the endothelial cells of the continuous endothelium limit paracellular passage of macromolecular therapeutics. Transport across this barrier most likely occurs by transcytosis (35). Several plasma proteins, such as albumin, transferrin, immunoglobulins, and lipoproteins, are transported across the continuous endothelium by caveolin-mediated transcytosis (36). In fact, approximately $60 \%$ of the body's total albumin resides in the tissue interstitium of skeletal muscle, skin, and other tissues at any given time (37). Thus, interactions with plasma proteins may enable nucleic acid drug delivery into tissues such as skeletal muscle and heart (38). It should be noted that the bloodbrain barrier (BBB) represents a special type of continuous endothelium, further limiting passage of nucleic acid drugs.

Cellular entry. Upon entry into the tissue interstitium and arrival at the cell surface of interest, nucleic acid drugs must adhere to cell surface proteins, which can assist their cellular entry via endocytic processes (Figure 2 and ref. 4). Unmodified single- and double-stranded nucleic acids have poor cell uptake properties because of their inability to adhere to cell surface proteins (39). Cell uptake of single-stranded nucleic acids can be augmented by the PS modification, which enhances avidity for proteins, including cell surface proteins (40). Duplexes of PS oligonucleotides can result in a substantial loss of binding affinity for cell surface proteins $(22,41)$. Presumably, the flexible nature of single-stranded nucleic acids facilitates interactions of the hydrophobic/anionic sulfur atom of the PS backbone and the amphipathic nucleobases with protein surfaces. In contrast, double-stranded nucleic acids are more rigid and do not expose the nucleobases for efficient interactions with protein surfaces (42). Alternately, attaching hydrophobic moieties to single- and double-stranded nucleic acids can enhance association with plasma lipoproteins, which can facilitate uptake by lipoprotein uptake receptors such as SR-BI or $\operatorname{LDLR}(43,44)$. The cell uptake properties of nucleic acid drugs can also be enhanced by conjugation of ligands for specific receptor systems, which can promote uptake by targeted delivery (45).

Escape from endosomal vesicles. The major pathway for cellular entry appears to be endocytosis. Once endocytosed into early endosomes, the nucleic acid drug is within the interior of the plasma membrane boundary and thus "inside" of the cell 


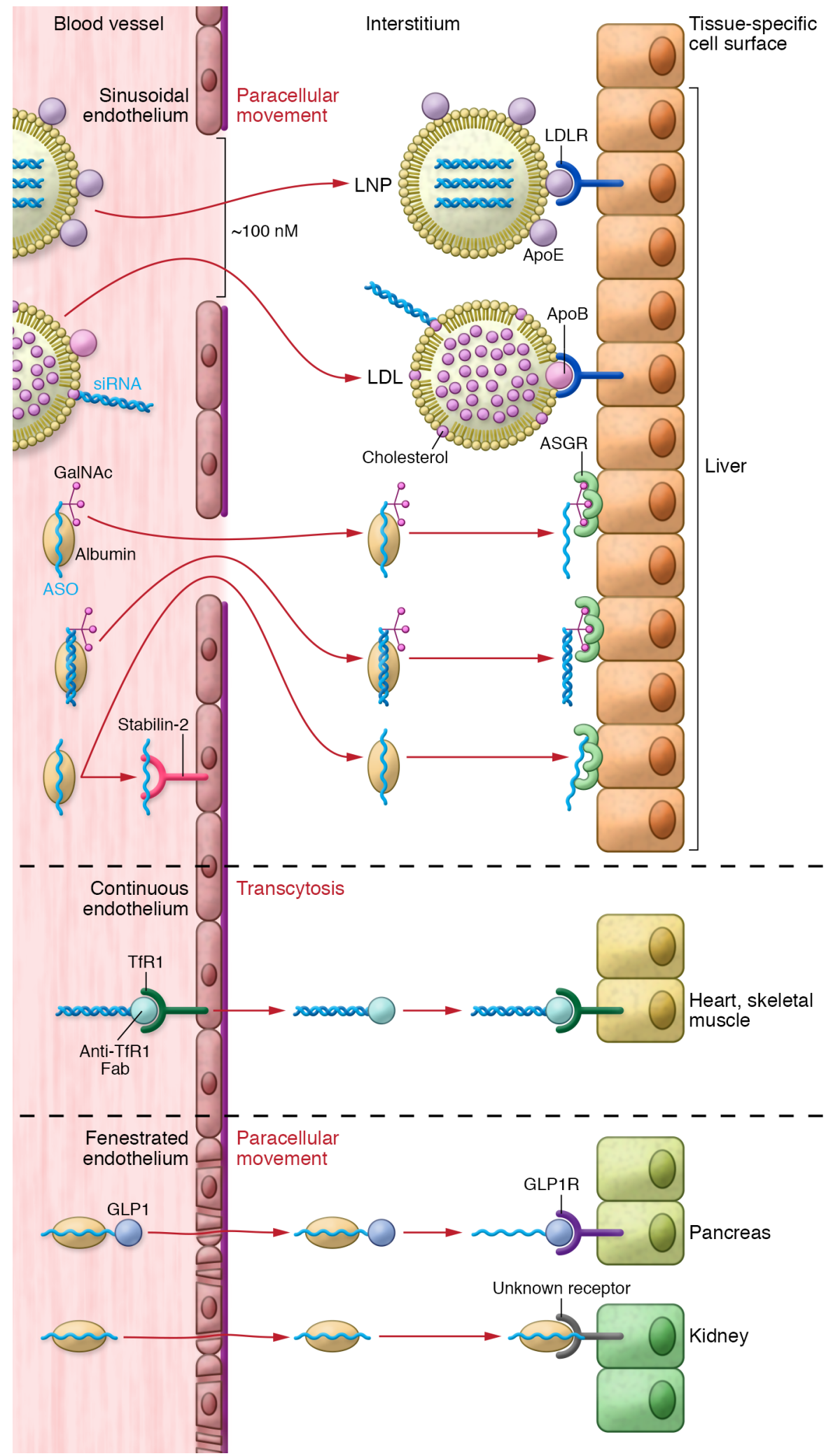

Figure 3. The tissue and cellular barriers an oligonucleotide drug must overcome for effective delivery. Interaction with plasma proteins facilitates distribution to peripheral tissues from the site of injection. Oligonucleotide drugs can gain access to the tissue interstitium by paracellular or transcellular transport across the capillary endothelium. Upon arrival at the cell surface of interest, oligonucleotide drugs can gain cellular entry by interaction with the targeted cell surface receptor.

that osmotic pressure caused by "proton sponging" or $\mathrm{pH}$-dependent lipid transitions compromise endosomal integrity or induce rupture, leading to release of the drug into cytoplasm (46-48). Unformulated oligonucleotide drugs possess no known mechanism to transit into the cytosol yet obviously accomplish this feat, given their clinically proven ability to modulate the RNA target in the intended tissue. How these oligonucleotides accomplish this topological conversion ("endosomal escape") is a fascinating question relatively shrouded in mystery.

\section{Strategies to overcome barriers to activity}

Effective delivery of nucleic acid drugs to cells and tissues of interest requires delivery strategies that can overcome the challenges enumerated above (Figure 3). In this section, we will highlight formulation and chemical modification strategies that have been successfully used to deliver nucleic acid drugs in animals or in the clinic. For ease of comprehension, this section is divided into broad categories of strategies focused on (a) formulation, (b) chemical promotion of interactions with plasma proteins, and (c) chemical promotion of cell surface interactions.

\section{Formulation strategies for single-stranded and} double-stranded oligonucleotide delivery

Lipid nanoparticles for delivery of siRNA to liver. Strategies based on lipid nanoparticles (LNPs) have effectively delivered nonstabilized or minimally stabilized siRNA to the liver (49). LNP-encapsulated siRNAs are approximately $100 \mathrm{~nm}$ in diameter and have neutral surface charge, which allows effec-

in one technical sense. From a topological perspective, however, the oligonucleotide resides in the extracellular space and must still cross a lipid bilayer to gain access to its RNA target in the cytosol/nucleus. The leading models for drugs formulated with varying kinds of polymers or cationic lipids suggest tive delivery to the liver parenchyma via the sinusoidal fenestrae. Delivery to nonphagocytic cell types and tissues other than BM is limited (50), as these particles do not cross the intact endothelium. LNPs can acquire apolipoprotein $\mathrm{E}$ from plasma in their corona, which actively directs them into hepatocytes via LDLR (51). Upon 
Table 2. Cell surface receptors involved in uptake of nucleic acid drugs

\begin{tabular}{|c|c|c|c|c|c|c|}
\hline Nucleic acid cargo & Ligand & Receptor & Class & Tissue & Cell type & Refs. \\
\hline ASO & PS & Stabilin-1 and -2 & Lectin/scavenger & Liver, spleen, lymphatics, BM & Endothelial cells & 68 \\
\hline ASO & PS & ASGR & Lectin & Liver & Hepatocytes & 39,70 \\
\hline ASO & PS & SRA & Scavenger & Liver & Kupffer cells & 113 \\
\hline ASO & PS & EGFR & RTK & Liver & Hepatocytes & 114 \\
\hline ASO, siRNA & Chol./LDL & LDLR & LDLR & Liver & Multiple & $44,51,62$ \\
\hline ASO, siRNA & Chol./HDL & SR-BI & Scavenger & Multiple & Multiple & 44 \\
\hline
\end{tabular}

ASGR, asialoglycoprotein receptor; RTK, receptor tyrosine kinase; SRA, Scavenger receptor A.

internalization and transfer into late endosomal compartments, the change in endosomal $\mathrm{pH}$ causes the cationic lipids in the LNP to undergo phase transition, forming an inverted hexagonal phase, a nonbilayer lipid structure that induces membrane permeability and LNP disintegration $(46,47,52)$. Patisiran (trade name Onpattro), an LNP-formulated siRNA that showed excellent results for reducing hepatic expression of mutant transthyretin (TTR) gene (53), was approved by the FDA for the treatment of polyneuropathy of hereditary transthyretin-mediated amyloidosis in adults.

Vitamin A-modified nanoparticles were used to deliver siRNA to stellate cells for the treatment of liver fibrosis (54). Inserting vitamin $\mathrm{A}$ in the liposomal formulation led to incorporation of retinol-binding protein (RBP) in the corona, which facilitated uptake by the RBP receptor expressed on activated hepatic stellate cells. The vitamin A-modified liposomal formulation containing siRNA against HSP47 showed excellent resolution of hepatic cirrhosis in three different in vivo models and is currently in clinical trials.

Spherical nucleic acids for delivery to macrophages. Spherical nucleic acids (SNAs) are distinct from liposome- or nanoparticle-encapsulated nucleic acids in that they resemble micelles with DNA forming the corona and a gold nanoparticle or liposomal core (55). They can be smaller in diameter (20-70 nm) than LNP formulations and can be prepared using single- or double-stranded oligonucleotides. Like most nanoparticulate formulations, SNAs are taken up preferentially by phagocytic cells like macrophages and keratinocytes. SNAs have shown interesting properties for dermal delivery of oligonucleotide therapeutics and were advanced into the clinic for treatment of psoriasis (56) and other indications (57). SNAs have also been used to create nucleic acid displays with CpG DNA to activate TLRs for immune-stimulatory applications and for solubilizing poorly soluble anticancer therapeutics (58).

\section{Promoting plasma protein interaction and peripheral tissue distribution}

Cholesterol-conjugated siRNA. Double-stranded nucleic acids, even those with PS and 2'-sugar modifications, show poor activity in mice (59). Conjugating siRNA to hydrophobic moieties such as cholesterol has been effective in promoting association with plasma lipoproteins and achieving tissue distribution and cellular uptake (44). Association with LDL or its protein component apolipoprotein B promotes hepatic uptake via LDLR. In contrast, association with HDL or apolipoprotein E promotes uptake into peripheral tissues via SR-BI. While the majority of work with cho-
lesterol-siRNA conjugates was directed toward hepatic delivery, recent work demonstrated that cholesterol conjugation can deliver siRNA to muscle and other extrahepatic tissues in mice $(60,61)$.

Tocopherol-conjugated ASO-RNA heteroduplexes. Yokota and colleagues recently investigated the use of tocopherol-conjugated ASO-RNA heteroduplexes for enhancing ASO potency in the liver (62). Single-stranded PS ASOs generally associate with albumin, but this interaction is lost in double-stranded designs. Thus, the tocopherol moiety was conjugated to the RNA strand of the ASO heteroduplex to enhance association with serum lipoproteins, and this design showed 7- to 10-fold improved potency in the liver. The tocopherol-ASO duplex also showed reduced activity in an LDLR-KO mouse, suggesting that this receptor was involved in the uptake of the lipoprotein-bound ASO duplex.

Single-stranded PS nucleic acids. Single-stranded PS-modified ASOs bind to plasma proteins such as albumin, which facilitates their distribution to tissues peripheral from the site of injection (63). They distribute broadly, except across the BBB, with tissues such as kidney, liver, spleen, LNs, and BM showing the highest accumulation. Sixteen- to 20-mer PS oligonucleotides associate with albumin, with binding constants in the $10-50 \mu \mathrm{M}$ range. RNase $\mathrm{H}$-dependent ASOs showed 2-fold enhanced potency in $\alpha_{2}$-macroglobin-KO mice, suggesting that association with proteins such as $\alpha_{2}$-macroglobin may steer the ASO toward less productive cellular or tissue compartments (64). The reversible association of single-stranded PS nucleic acids with plasma proteins allows the oligonucleotide to partition onto cell surface proteins, which promotes entry into cells and tissues.

\section{Chemical strategies to promote binding to cell surface proteins}

PS modification to enhance binding to cell surface proteins. Almost all mammalian cells can internalize PS-modified nucleic acids in an energy-dependent manner. The protein-binding properties of single-stranded PS nucleic acids facilitate interactions with acceptor proteins on the cell surface. Once accomplished, endocytic processes internalize the adsorbed nucleic acid into the cell. Earlier studies estimated 200,000 binding sites for PS ASOs on the surface of K562 cells (40) and showed that PS ASOs interact with heparin-binding proteins in the extracellular matrix (65). However, the identity of these cell surface acceptors remained largely unknown. In this section, we highlight cell surface proteins involved in the uptake of single-stranded PS oligonucleotides in tissues such as the liver, spleen, LNs, and BM (summarized in Table 2). 
Table 3. Receptor-ligand pairs for cell-specific delivery of nucleic acid drugs

\begin{tabular}{|c|c|c|c|c|c|c|}
\hline Nucleic acid cargo & Ligand & Receptor & Class & Tissue & Cell type & Refs. \\
\hline ASO, siRNA, anti-miR & GalNAc & ASCR & Lectin & Liver & Hepatocytes & 89,91 \\
\hline siRNA & Anti-TfR Fab & TfR1 & Nutrient & Skeletal muscle, heart & Muscle cells & 101 \\
\hline ASO & Anti-CD22 antibody & $\mathrm{CD} 22$ & Lectin & Immune & B cells & 117,118 \\
\hline
\end{tabular}

Single-stranded PS oligonucleotides can interact with the stabilin class of scavenger receptors (66), which are highly expressed in the spleen, liver, LNs, and BM (67) - tissues corresponding to sites of higher accumulation of PS ASOs (25). The stabilins bind and internalize large polyanionic components of the extracellular matrix like hyaluronic acid, chondroitin and dermatan sulfates, and other glycosaminoglycans, suggesting a common pharmacophore with polyanionic PS ASOs (68). HEK293 cells expressing stabilin-2 showed 3- to 5-fold enhanced uptake of single-stranded PS ASOs (66). This resulted in a 10- to 20-fold enhancement in antisense activity in these cells compared with wild-type HEK cells. Only single-stranded PS oligonucleotides were able to bind the purified ectodomain of stabilin-2, while duplexes of the corresponding oligonucleotides showed no interaction (22). Stabilin-2KO mice showed no PS ASO accumulation in sinusoidal cells in the liver or spleen. In wild-type mice, these cells show high stabilin expression and PS ASO accumulation, highlighting the role of the stabilin receptors in PS ASO uptake.

Asialoglycoprotein receptor (ASGR) is highly expressed on hepatocytes in the liver (69). PS ASOs were shown to interact with ASGR membranes in a length-, charge-, and PS-dependent manner in a competition binding assay (41). Cell lines that express ASGR1 (constitutively or inducibly) showed enhanced uptake and antisense activity for PS ASOs relative to control HEK293 cells (39). PS ASOs showed 2- to 3-fold reduced antisense activity in the liver in ASGR-KO mice, suggesting that this receptor has a role in uptake of PS ASOs into liver hepatocytes (70).

Cell-specific delivery using receptor-ligand systems. This section highlights select receptor-ligand pairs reported recently for the effective delivery of therapeutic nucleic acids in animals or in the clinic (Figure 3 and Table 3). A more comprehensive review of delivery of therapeutic oligonucleotides can be found in ref. 71 .

ASGR is a C-type lectin abundantly ( $\sim 500,000$ copies per cell) (72) expressed in hepatocytes $(73,74)$. ASGR clusters in coated pits on the basolateral surface of hepatocytes and is internalized by clathrin-mediated endocytosis $(75,76)$. The receptor-ligand complex is delivered to endosomal compartments, where acidification leads to dissociation of the complex. The soluble ligand is transported to lysosomes for degradation, while the membrane-bound receptor is recycled back to the cell surface, where it can participate in multiple rounds of endocytosis (77).

Baenziger's group showed that ASGR binds glycoproteins terminating with sialic acid $\alpha 2,6$-galactose and $N$-acetylgalactosamine (GalNAc) sugars and found elevated levels of these glycoproteins in blood from ASGR-KO mice $(78,79)$. They postulated that ASGR acts as a buffer to maintain the physiological levels of its glycoprotein ligands, several of which circulate in blood at concentrations close to their dissociation constant for the receptor. Grewal et al. later showed that ASGR's enormous clearance capacity enables rapid clearance of platelets desialylated by bacterial neuraminidases (80).

The functional ASGR comprises two subunits that form a heterooligomeric complex in varying ratios (2:1 to 5:1, ASGR1/ASGR2) $(69,81)$. The ASGR1 subunit possesses the carbohydrate recognition domain and the cytoplasmic signal to interact with clathrin adaptors in coated pits $(82,83)$. The physiological role of the ASGR2 subunit is unclear and may serve to cluster the ASGR1 subunit on the cell surface. Elegant work by Lee and colleagues showed that synthetic glycosides with branched tethers bind ASGR with high affinity $(84,85)$. Binding affinity was dependent on the nature of the sugar (GalNAc $>$ galactose), the number of sugars $(4=3>2>1)$, and the geometrical spacing between sugar moieties (86). The x-ray crystal structure of the ASGR1 ectodomain shows that the carbohydrate binding pocket is shallow and solvent-exposed (87). Consequently, monovalent sugar ligands do not display high binding affinity for ASGR, and multiple interactions with the oligomeric receptor are required to enhance avidity (88).

Manoharan's group showed that chemically modified siRNA duplexes can be effectively delivered to hepatocytes by targeting of ASGR using trivalent GalNAc conjugates (89). The GalNAcsiRNA conjugates were rapidly cleared from plasma into hepatocytes, and and excess GalNAc ligand could compete with this uptake, showing specificity of ASGR-mediated uptake. Recent studies highlighted the importance of chemical modification to the GalNAc-siRNA conjugates to enhance potency and duration of effect (90). Chemical modifications help siRNA survive nucleolytic degradation as it transits through endo-lysosomal compartments before release to the cytosol. Several GalNAc-siRNAs are currently in clinical development and have shown robust activity for reducing gene expression in hepatocytes in rodents, nonhuman primates, and humans (Table 4). This technology represents one of the first clinical demonstrations of receptor-mediated delivery enhancing the efficacy of nucleic acid-based therapeutics.

GalNAc conjugates have also been used to deliver singlestranded ASO therapeutics to hepatocytes (91). Switching the distribution of PS ASOs from nonparenchymal cells (NPCs) to hepatocytes via ASGR-mediated delivery resulted in 10-fold increased potency in rodents. The improved potency of GalNAc-ASO therapeutics has translated to the clinic, where 30 -fold potency enhancement relative to unconjugated ASOs was observed (9). 
Table 4. GaINAc-conjugated nucleic acid therapeutics in clinical development

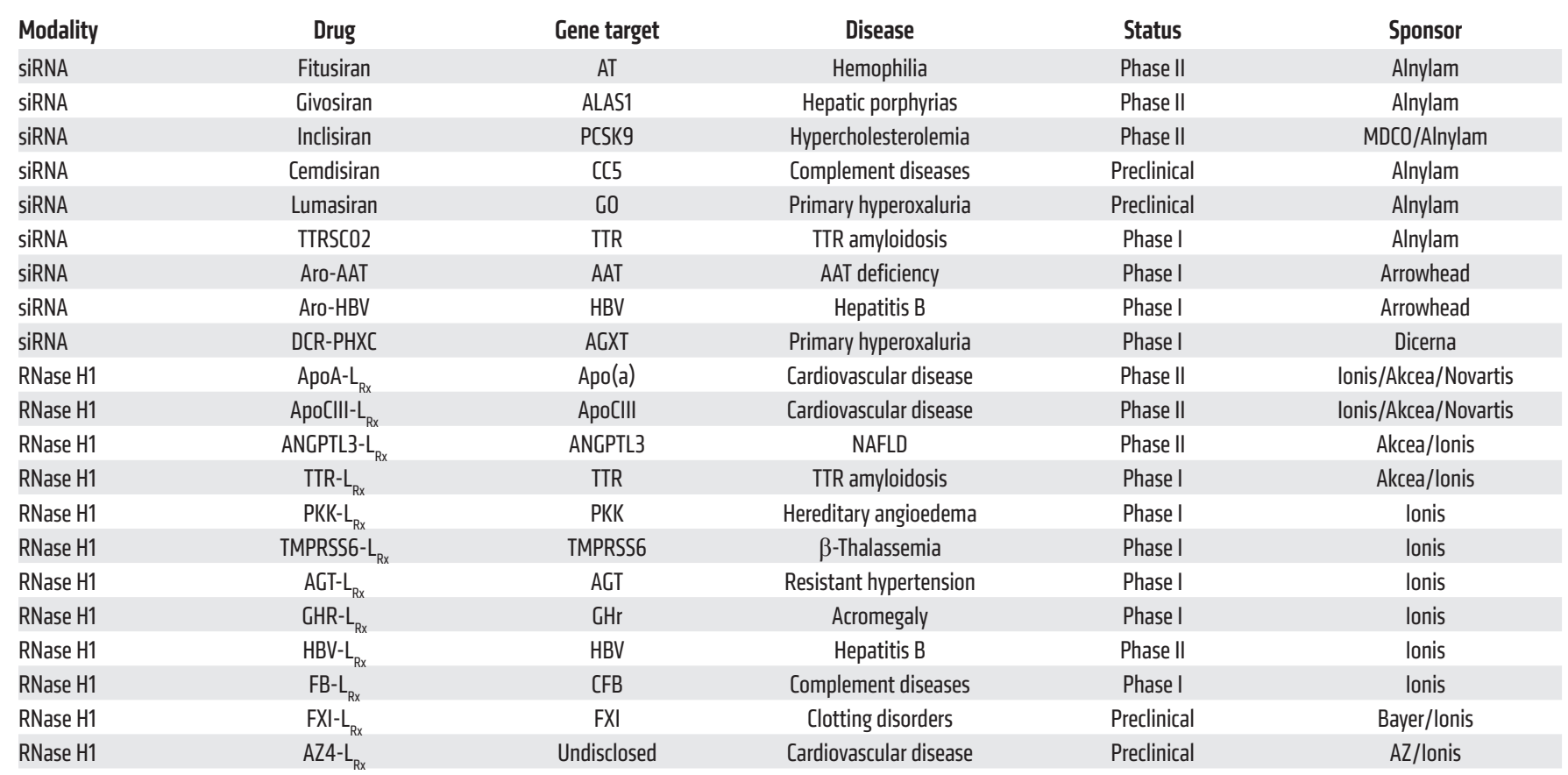

MDCO, The Medicines Company; NAFLD, nonalcoholic fatty liver disease.

Structure-activity relationships of GalNAc-conjugated siRNA showed that trivalent GalNAc sugars are optimal for ASGR binding and for enhancing potency $(92,93)$. Positioning of the cluster near the ends of the siRNA was preferred, and incorporating sugars at positions where they could interfere with RISC loading reduced potency. In contrast, two GalNAc sugars attached to the same or opposite ends of a single-stranded ASO were sufficient for optimal receptor binding and potency enhancement (41). Even a single GalNAc sugar was sufficient to enhance ASO potency 5-fold (94). Evaluation of mono GalNAc-ASO conjugates in a competition binding assay revealed that the ASO contributes to receptor binding in a length-, charge-, and PS-dependent manner (41). These results challenged the existing model for ASGR binding, which emphasizes the sugar's distance from the branchpoint and the relative spatial orientation between the sugar moieties. Further investigations using engineered cells revealed that the ASGR2 subunit was dispensable and that the ASGR1 subunit is sufficient for internalizing the nucleic acid cargo (39).

Glucagon-like peptide 1 receptor (GLP1R) is a secretin-like, class B GPCR primarily expressed in pancreatic $\beta$ cells, with limited expression in other tissues such as the brain and gut $(95,96)$. Exenatide shares significant sequence homology to GLP1 and is a potent GLP1R agonist but is resistant to DPP-4-mediated metabolism (97). Several GLP1/exendin-4 derivatives have since emerged in the clinic (98), and GLP1R agonism has become an important treatment for type 2 diabetes.

The pancreatic islets of Langerhans are especially insensitive to uptake of PS ASOs (99). Finan showed that a modified GLP1 peptide can deliver estrogens to $\beta$ cells (100), and this strategy was recently investigated for PS ASO delivery. ASO delivery via GLP1R requires conjugation to an agonist ligand, which induces receptor activation and internalization. GLP1-ASO conjugates were selectively internalized into GLP1R-expressing HEK293 cells, thereby producing a 40-fold enhancement in ASO potency over the unconjugated counterpart (101). Enhanced activity was also demonstrated in isolated pancreatic islets. More strikingly, accumulation and activity of unconjugated ASOs was undetectable in $\beta$ cells following systemic administration, but the GLP1-ASO conjugates showed dose-dependent accumulation and potent knockdown of gene expression. Potentiation of activity was not observed in GLP1R-KO mice, confirming that ASO delivery indeed occurs via GLP1R.

Transferrin receptor 1 (TfR1; also known as CD71), like ASGR and LDLR, is an extensively studied model receptor-ligand system and has provided considerable insight into the cellular properties and mechanisms of nutrient/scavenger receptor cargo internalization and endocytic sorting (102). Similarly to ASGR and LDLR, TfR1 undergoes constitutive endocytosis and recycling to the plasma membrane and possesses $\mathrm{pH}$-dependent ligand binding to enable proper sorting of endocytosed cargo. Unlike with ASGR and LDLR, however, the relevant cargo $\left(\mathrm{Fe}^{+3}\right)$ does not bind to the receptor itself but instead shows $\mathrm{pH}$-dependent association with TfR1's ligand, transferrin $(103,104)$. Comprehensive reviews of TfR1 biology can be found elsewhere (105).

Because of the nature of transferrin-TfR1 binding and sorting, exploiting TfR1 for targeted oligonucleotide delivery is more complex than exploiting ASGR. For TfR1-mediated delivery the cargo has to behave more like iron than transferrin, which is continuously recycled out of the cell rather than accumulated in the endocytic pathway. Moreover, transferrin is large $(\sim 77,000 \mathrm{Da})$ and not easily amenable to oligonucleotide conjugation. Anti-TfR1 antibodies, 
therefore, are the primary agents for TfR1 targeting of oligonucleotide therapeutics. The potential for TfR1 targeting was recently demonstrated, showing efficient and profound knockdown of gene expression in skeletal and cardiac muscle via systemically delivered anti-TfR1-Fab-siRNA conjugates in mice (106).

\section{Intracellular sorting and release from endosomal vesicles}

Sorting and release of PS ASOs. Multiple lines of evidence indicate that active sorting processes involving specific membrane trafficking factors and proteins may mediate endosomal escape. Furthermore, it is generally agreed that endosomal escape occurs from a late, prelysosomal compartment (i.e., the late endosome), suggesting some level of specificity to the process. Gene silencing studies implicated multiple proteins associated with endosomal trafficking in endosomal escape and activity of oligonucleotides (42, 107-109). Most identified proteins regulate endocytosis, endosome maturation, and/or multivesicular body formation/function.

Small molecules that promote release from endo-lysosomes. It is estimated that a small $(<1 \%)$ proportion of internalized oligonucleotides escape the endosomal compartments through poorly understood mechanisms. A variety of strategies have been used to facilitate oligonucleotide release from endo-lysosomes. These include nucleic acid complexation with releasing agents or the use of exogenous small-molecule compounds that promote endosomal escape. One of the earliest examples of exogenous small-molecule escape potentiators is Retro-1. This compound was first identified in a screen for small-molecule inhibitors of retrograde transport of Shiga-like bacterial toxins, a prerequisite for their escape into the cytosolic space via the ER (110). Retro-1 was subsequently shown to enhance oligonucleotide activity by increasing escape from a late endocytic compartment (111). A high-throughput screen to identify novel compounds with similar cellular effects reported three distinct chemical scaffolds that enhanced oligonucleotide activity (112). The most potent compound, UNC7938, increased nuclear accumulation of a PS ASO that was accompanied by loss of ASO/Rab7 colocalization, indicating escape from a late endosomal compartment.

\section{Future directions}

Tissue targeting of macromolecules has been an active area of research for decades and is beginning to be realized in several therapeutic products. The recent results with GalNAc-conjugated oligonucleotide drugs are an example of the potential that tissue targeting can bring to the ASO field. There are many opportunities to translate advances in cell biology, molecular biology, receptor pharmacology, medicinal chemistry, and other scientific disciplines into more effective and better-tolerated ASO therapeutics. It will be important to diversify the ligands and acceptors that can be used to target ASO drugs, broadening the range of tissues that can be efficiently targeted. In addition, identifying efficient means for systemic ASO therapeutics to traverse protected tissue barriers such as the CNS will be an important area of continued investigation. Targeted delivery of antisense-based drugs will be an important avenue of research to propel the technology forward into the next stage of advancement.

Address correspondence to: C. Frank Bennett, Ionis Pharmaceuticals Inc., 2855 Gazelle Court, Carlsbad, California 92010, USA. Phone: 760.603.2336; Email: fbennett@ionisph.com.
1. Dan N, et al. Antibody-drug conjugates for cancer therapy: chemistry to clinical implications. Pharmaceuticals (Basel). 2018;11(2):E32.

2. Sau S, Alsaab HO, Kashaw SK, Tatiparti K, Iyer AK. Advances in antibody-drug conjugates: a new era of targeted cancer therapy. Drug Discov Today. 2017;22(10):1547-1556.

3. Crooke ST, Witztum JL, Bennett CF, Baker BF. RNA-targeted therapeutics. Cell Metab. 2018;27(4):714-739.

4. Crooke ST, Wang S, Vickers TA, Shen W, Liang XH. Cellular uptake and trafficking of antisense oligonucleotides. Nat Biotechnol. 2017;35(3):230-237.

5. Bennett CF, Swayze EE. RNA targeting therapeutics: molecular mechanisms of antisense oligonucleotides as a therapeutic platform. Annu Rev Pharmacol Toxicol. 2010;50:259-293.

6. Nelson SF, Miceli MC. FDA approval of eteplirsen for muscular dystrophy. JAMA. 2017;317(14):1480.

7. Ottesen EW. ISS-N1 makes the first FDAapproved drug for spinal muscular atrophy. Transl Neurosci. 2017;8:1-6.

8. Wilson RC, Doudna JA. Molecular mechanisms of RNA interference. Annu Rev Biophys. 2013;42:217-239.

9. Viney NJ, et al. Antisense oligonucleotides targeting apolipoprotein(a) in people with raised lipoprotein(a): two randomised, double-blind, placebo-controlled, dose-ranging trials. Lancet.
2016;388(10057):2239-2253.

10. Zimmermann TS, et al. Clinical proof of concept for a novel hepatocyte-targeting GalNAc-siRNA conjugate. Mol Ther. 2017;25(1):71-78.

11. Wan WB, Seth PP. The medicinal chemistry of therapeutic oligonucleotides. J Med Chem. 2016;59(21):9645-9667.

12. Krieg AM. CpG motifs in bacterial DNA and their immune effects. Annu Rev Immunol. 2002;20:709-760.

13. Takahasi K, et al. Nonself RNA-sensing mechanism of RIG-I helicase and activation of antiviral immune responses. Mol Cell. 2008;29(4):428-440.

14. Obika S, et al. Synthesis of 2'-O,4'-C-methyleneuridine and -cytidine. Novel bicyclic nucleosides having a fixed $\mathrm{C}_{3}$, -endo sugar puckering. Tetrahedron Lett. 1997;38(50):8735-8738.

15. Koshkin AA, Nielsen P, Meldgaard M, Rajwanshi VK, Singh SK, Wengel J. LNA (Locked Nucleic Acid): an RNA mimic forming exceedingly stable LNA:LNA suplexes. JAm Chem Soc. 1998;120(50):13252-13253.

16. Seth PP, et al. Synthesis and biophysical evaluation of 2',4'-constrained 2'O-methoxyethyl and 2',4'-constrained 2'O-ethyl nucleic acid analogues. JOrg Chem. 2010;75(5):1569-1581.

17. Pallan PS, et al. Structure and nuclease resistance of 2',4'-constrained 2'-O-methoxyethyl (cMOE) and 2'-O-ethyl (cEt) modified DNAs. Chem Commun (Camb). 2012;48(66):8195-8197.
18. Summerton J, Weller D. Morpholino antisense oligomers: design, preparation, and properties. Antisense Nucleic Acid Drug Dev. 1997;7(3):187-195.

19. Eckstein F. Phosphorothioates, essential components of therapeutic oligonucleotides. Nucleic Acid Ther. 2014;24(6):374-387.

20. Brown DA, et al. Effect of phosphorothioate modification of oligodeoxynucleotides on specific protein binding. J Biol Chem. 1994;269(43):26801-26805.

21. Dertinger D, Behlen LS, Uhlenbeck OC. Using phosphorothioate-substituted RNA to investigate the thermodynamic role of phosphates in a sequence specific RNA-protein complex. Biochemistry. 2000;39(1):55-63.

22. Gaus H, Miller CM, Seth PP, Harris EN. Structural determinants for the interactions of chemically modified nucleic acids with the stabilin-2 clearance receptor. Biochemistry. 2018;57(14):2061-2064.

23. Manoharan M. 2'-Carbohydrate modifications in antisense oligonucleotide therapy: importance of conformation, configuration and conjugation. Biochim Biophys Acta. 1999;1489(1):117-130.

24. Jayaraman M, et al. Maximizing the potency of siRNA lipid nanoparticles for hepatic gene silencing in vivo. Angew Chem Int Ed Engl. 2012;51(34):8529-8533.

25. Geary RS, Norris D, Yu R, Bennett CF. Pharmacokinetics, biodistribution and cell uptake of 
antisense oligonucleotides. Adv Drug Deliv Rev 2015;87:46-51.

26. Tavitian $B$, et al. In vivo imaging of oligonucleotides with positron emission tomography. Nat Med.1998;4(4):467-471.

27. Nedredal GI, Elvevold KH, Ytrebø LM, Olsen R, Revhaug A, Smedsrød B. Liver sinusoidal endothelial cells represents an important blood clearance system in pigs. Comp Hepatol. 2003;2(1):1.

28. Kawai Y, Smedsrød B, Elvevold K, Wake K. Uptake of lithium carmine by sinusoidal endothelial and Kupffer cells of the rat liver: new insights into the classical vital staining and the reticulo-endothelial system. Cell Tissue Res. 1998;292(2):395-410.

29. Murphy JE, Tedbury PR, Homer-Vanniasinkam S, Walker JH, Ponnambalam S. Biochemistry and cell biology of mammalian scavenger receptors. Atherosclerosis. 2005;182(1):1-15.

30. Suk JS, Xu Q, Kim N, Hanes J, Ensign LM. PEGylation as a strategy for improving nanoparticle-based drug and gene delivery. Adv Drug Deliv Rev. 2016;99(pt A):28-51.

31. Campbell F, et al. Directing nanoparticle biodistribution through evasion and exploitation of Stab2-dependent nanoparticle uptake. ACS Nano. 2018;12(3):2138-2150.

32. Kolka CM, Bergman RN. The barrier within: endothelial transport of hormones. Physiology (Bethesda). 2012;27(4):237-247.

33. Risau W. Development and differentiation of endothelium. Kidney Int Suppl. 1998;67:S3-S6.

34. Zhang YN, Poon W, Tavares AJ, McGilvray ID, Chan WCW. Nanoparticle-liver interactions: Cellular uptake and hepatobiliary elimination. JControl Release. 2016;240:332-348.

35. Simionescu M, Gafencu A, Antohe F. Transcytosis of plasma macromolecules in endothelial cells: a cell biological survey. Microsc Res Tech. 2002;57(5):269-288.

36. Schnitzer JE. Caveolae: from basic trafficking mechanisms to targeting transcytosis for tissue-specific drug and gene delivery in vivo. Adv Drug Deliv Rev. 2001;49(3):265-280.

37. Ellmerer $\mathrm{M}$, et al. Measurement of interstitial albumin in human skeletal muscle and adipose tissue by open-flow microperfusion. Am J Physiol Endocrinol Metab. 2000;278(2):E352-E356.

38. Larsen MT, Kuhlmann M, Hvam ML, Howard KA. Albumin-based drug delivery: harnessing nature to cure disease. Mol Cell Ther. 2016;4:3.

39. Tanowitz M, Hettrick L, Revenko A, Kinberger GA, Prakash TP, Seth PP. Asialoglycoprotein receptor 1 mediates productive uptake of $\mathrm{N}$-acetylgalactosamine-conjugated and unconjugated phosphorothioate antisense oligonucleotides into liver hepatocytes. Nucleic Acids Res. 2017;45(21):12388-12400.

40. Beltinger C, et al. Binding, uptake, and intracellular trafficking of phosphorothioate-modified oligodeoxynucleotides. JClin Invest. 1995;95(4):1814-1823.

41. Schmidt K, et al. Characterizing the effect of GalNAc and phosphorothioate backbone on binding of antisense oligonucleotides to the asialoglycoprotein receptor. Nucleic Acids Res. 2017;45(5):2294-2306.

42. Koller E, Vincent TM, Chappell A, De S, Manoha- ran M, Bennett CF. Mechanisms of single-stranded phosphorothioate modified antisense oligonucleotide accumulation in hepatocytes. Nucleic Acids Res. 2011;39(11):4795-4807.

43. Crooke ST, et al. Pharmacokinetic properties of several novel oligonucleotide analogs in mice. JPharmacol Exp Ther. 1996;277(2):923-937.

44. Wolfrum C, et al. Mechanisms and optimization of in vivo delivery of lipophilic siRNAs. Nat Biotechnol. 2007;25(10):1149-1157.

45. Juliano RL, Ming X, Carver K, Laing B. Cellular uptake and intracellular trafficking of oligonucleotides: implications for oligonucleotide pharmacology. Nucleic Acid Ther. 2014;24(2):101-113.

46. Zelphati O, Szoka FC. Mechanism of oligonucleotide release from cationic liposomes. Proc Natl Acad Sci U S A. 1996;93(21):11493-11498.

47. Gujrati M, Malamas A, Shin T, Jin E, Sun Y, Lu ZR. Multifunctional cationic lipid-based nanoparticles facilitate endosomal escape and reduction-triggered cytosolic siRNA release. Mol Pharm. 2014;11(8):2734-2744.

48. Freeman EC, Weiland LM, Meng WS. Modeling the proton sponge hypothesis: examining proton sponge effectiveness for enhancing intracellular gene delivery through multiscale modeling. J Biomater Sci Polym Ed. 2013;24(4):398-416.

49. Semple SC, et al. Rational design of cationic lipids for siRNA delivery. Nat Biotechnol. 2010;28(2):172-176.

50. Zhang G, et al. A delivery system targeting bone formation surfaces to facilitate RNAi-based anabolic therapy. Nat Med. 2012;18(2):307-314.

51. Akinc A, et al. Targeted delivery of RNAi therapeutics with endogenous and exogenous ligand-based mechanisms. Mol Ther. 2010;18(7):1357-1364.

52. Leung AK, et al. Lipid nanoparticles containing siRNA synthesized by microfluidic mixing exhibit an electron-dense nanostructured core. J Phys Chem C Nanomater Interfaces. 2012;116(34):18440-18450.

53. Suhr OB, et al. Efficacy and safety of patisiran for familial amyloidotic polyneuropathy: a phase II multi-dose study. Orphanet JRare Dis. 2015;10:109.

54. Sato Y, et al. Resolution of liver cirrhosis using vitamin A-coupled liposomes to deliver siRNA against a collagen-specific chaperone. Nat Biotechnol. 2008;26(4):431-442.

55. Chinen AB, Guan CM, Ko CH, Mirkin CA. The impact of protein corona formation on the macrophage cellular uptake and biodistribution of spherical nucleic acids. Small. 2017;13(16):10.1002/smll.201603847.

56. Zheng D, et al. Topical delivery of siRNA-based spherical nucleic acid nanoparticle conjugates for gene regulation. Proc Natl Acad Sci US A. 2012;109(30):11975-11980.

57. Kapadia CH, Melamed JR, Day ES. Spherical nucleic acid nanoparticles: therapeutic potential. BioDrugs. 2018;32(4):297-309.

58. de Vries JW, Zhang F, Herrmann A. Drug delivery systems based on nucleic acid nanostructures. J Control Release. 2013;172(2):467-483.

59. Soutschek J, et al. Therapeutic silencing of an endogenous gene by systemic administration of modified siRNAs. Nature. 2004;432(7014):173-178.

60. Khan T, et al. Silencing myostatin using choles- terol-conjugated sirnas induces muscle growth. Mol Ther Nucleic Acids. 2016;5(8):e342.

61. Osborn MF, et al. Hydrophobicity drives the systemic distribution of lipid-conjugated siRNAs via lipid transport pathways [published online ahead of print December 8, 2018]. Nucleic Acids Res. https://doi.org/10.1093/nar/gky1232.

62. Nishina K, et al. DNA/RNA heteroduplex oligonucleotide for highly efficient gene silencing. Nat Commun. 2015;6:7969.

63. Geary RS. Antisense oligonucleotide pharmacokinetics and metabolism. Expert Opin Drug Metab Toxicol. 2009;5(4):381-391.

64. Shemesh CS, et al. Pharmacokinetic and pharmacodynamic investigations of ION-353382, a model antisense oligonucleotide: using alpha-2-macroglobulin and murinoglobulin double-knockout mice. Nucleic Acid Ther. 2016;26(4):223-235.

65. Guvakova MA, Yakubov LA, Vlodavsky I, Tonkinson JL, Stein CA. Phosphorothioate oligodeoxynucleotides bind to basic fibroblast growth factor, inhibit its binding to cell surface receptors, and remove it from low affinity binding sites on extracellular matrix. J Biol Chem. 1995;270(6):2620-2627.

66. Miller CM, et al. Stabilin-1 and Stabilin-2 are specific receptors for the cellular internalization of phosphorothioate-modified antisense oligonucleotides (ASOs) in the liver. Nucleic Acids Res. 2016;44(6):2782-2794.

67. Hare AK, Harris EN. Tissue-specific splice variants of HARE/Stabilin-2 are expressed in bone marrow, lymph node, and spleen. Biochem Biophys Res Commun. 2015;456(1):257-261.

68. Miller CM, et al. Receptor-mediated uptake of phosphorothioate antisense oligonucleotides in different cell types of the liver. Nucleic Acid Ther. 2018;28(3):119-127.

69. Stockert RJ. The asialoglycoprotein receptor: relationships between structure, function, and expression. Physiol Rev. 1995;75(3):591-609.

70. Donner AJ, et al. Co-administration of an excipient oligonucleotide helps delineate pathways of productive and nonproductive uptake of phosphorothioate antisense oligonucleotides in the liver. Nucleic Acid Ther. 2017;27(4):209-220.

71. Juliano RL. The delivery of therapeutic oligonucleotides. Nucleic Acids Res. 2016;44(14):6518-6548.

72. Schwartz AL, Rup D, Lodish HF. Difficulties in the quantification of asialoglycoprotein receptors on the rat hepatocyte. J Biol Chem. 1980;255(19):9033-9036.

73. Pricer WE, Ashwell G. The binding of desialylated glycoproteins by plasma membranes of rat liver. J Biol Chem. 1971;246(15):4825-4833.

74. Hudgin RL, Pricer WE, Ashwell G, Stockert RJ, Morell AG. The isolation and properties of a rabbit liver binding protein specific for asialoglycoproteins. J Biol Chem. 1974;249(17):5536-5543.

75. Wall DA, Wilson G, Hubbard AL. The galactose-specific recognition system of mammalian liver: the route of ligand internalization in rat hepatocytes. Cell. 1980;21(1):79-93.

76. Wall DA, Hubbard AL. Galactose-specific recognition system of mammalian liver: receptor distribution on the hepatocyte cell surface. J Cell Biol. 1981;90(3):687-696. 
77. Geuze HJ, Slot JW, Strous GJ, Lodish HF, Schwartz AL. Intracellular site of asialoglycoprotein receptor-ligand uncoupling: double-label immunoelectron microscopy during receptormediated endocytosis. Cell. 1983;32(1):277-287.

78. Park EI, Mi Y, Unverzagt C, Gabius HJ, Baenziger JU. The asialoglycoprotein receptor clears glycoconjugates terminating with sialic acid alpha 2,6GalNAc. Proc Natl Acad Sci U S A. 2005;102(47):17125-17129.

79. Steirer LM, Park EI, Townsend RR, Baenziger JU. The asialoglycoprotein receptor regulates levels of plasma glycoproteins terminating with sialic acid alpha2,6-galactose. J Biol Chem. 2009;284(6):3777-3783.

80. Grewal PK, et al. Inducing host protection in pneumococcal sepsis by preactivation of the Ashwell-Morell receptor. Proc Natl Acad Sci U S A. 2013;110(50):20218-20223.

81. Spiess M. The asialoglycoprotein receptor: a model for endocytic transport receptors. Biochemistry. 1990;29(43):10009-10018.

82. Geffen I, et al. Related signals for endocytosis and basolateral sorting of the asialoglycoprotein receptor. J Biol Chem. 1993;268(28):20772-20777.

83. Katzir Z, Nardi N, Geffen I, Fuhrer C, Henis YI. Dynamic interactions of the asialoglycoprotein receptor subunits with coated pits. Enhanced interactions of $\mathrm{H} 2$ following association with $\mathrm{H} 1$. J Biol Chem. 1994;269(34):21568-21575.

84. Ozaki K, Lee RT, Lee YC, Kawasaki T. The differences in structural specificity for recognition and binding between asialoglycoprotein receptors of liver and macrophages. Glycoconj J. 1995;12(3):268-274.

85. Lee RT, Lee YC. Facile synthesis of a high-affinity ligand for mammalian hepatic lectin containing three terminal $\mathrm{N}$-acetylgalactosamine residues. Bioconjug Chem. 1997;8(5):762-765.

86. Lee YC, Lee RT. In: Ernst B, Hart GW, Sinaý P eds. Interactions of oligosaccharides and glycopeptides with hepatic carbohydrate receptors. Carbohydrates in Chemistry and Biology. Weinheim, Germany: Wiley-VCH Verlag $\mathrm{GmbH} ; 2008: 549-561$.

87. Meier M, Bider MD, Malashkevich VN, Spiess M, Burkhard P. Crystal structure of the carbohydrate recognition domain of the $\mathrm{H} 1$ subunit of the asialoglycoprotein receptor. J Mol Biol. 2000;300(4):857-865.

88. Mammen M, Choi SK, Whitesides GM. Polyvalent interactions in biological systems: implications for design and use of multivalent ligands and inhibitors. Angew Chem Int Ed Engl. 1998;37(20):2754-2794.

89. Nair JK, et al. Multivalent N-acetylgalactosamineconjugated siRNA localizes in hepatocytes and elicits robust RNAi-mediated gene silencing. J Am
Chem Soc. 2014;136(49):16958-16961.

90. Nair JK, et al. Impact of enhanced metabolic stability on pharmacokinetics and pharmacodynamics of GalNAc-siRNA conjugates. Nucleic Acids Res. 2017;45(19):10969-10977.

91. Prakash TP, et al. Targeted delivery of antisense oligonucleotides to hepatocytes using triantennary $\mathrm{N}$-acetyl galactosamine improves potency 10 -fold in mice. Nucleic Acids Res. 2014;42(13):8796-8807.

92. Rajeev KG, et al. Hepatocyte-specific delivery of siRNAs conjugated to novel non-nucleosidic trivalent $\mathrm{N}$-acetylgalactosamine elicits robust gene silencing in vivo. Chembiochem. 2015;16(6):903-908.

93. Matsuda $\mathrm{S}$, et al. siRNA conjugates carrying sequentially assembled trivalent $\mathrm{N}$-acetylgalactosamine linked through nucleosides elicit robust gene silencing in vivo in hepatocytes. ACS Chem Biol. 2015;10(5):1181-1187.

94. Kinberger GA, et al. Conjugation of mono and di-GalNAc sugars enhances the potency of antisense oligonucleotides via ASGR mediated delivery to hepatocytes. Bioorg Med Chem Lett. 2016;26(15):3690-3693.

95. Al-Sabah S. Molecular pharmacology of the incretin receptors. Med Princ Pract. 2016;25(suppl 1):15-21.

96. Pyke C, et al. GLP-1 receptor localization in monkey and human tissue: novel distribution revealed with extensively validated monoclonal antibody. Endocrinology. 2014;155(4):1280-1290.

97. Briones M, Bajaj M. Exenatide: a GLP-1 receptor agonist as novel therapy for Type 2 diabetes mellitus. Expert Opin Pharmacother. 2006;7(8):1055-1064.

98. McBrayer DN, Tal-Gan Y. Recent advances in GLP-1 receptor agonists for use in diabetes mellitus. Drug Dev Res. 2017;78(6):292-299.

99. Hung G, et al. Characterization of target mRNA reduction through in situ RNA hybridization in multiple organ systems following systemic antisense treatment in animals. Nucleic Acid Ther. 2013;23(6):369-378.

100.Finan B, et al. Targeted estrogen delivery reverses the metabolic syndrome. Nat Med. 2012;18(12):1847-1856.

101. Ämmälä C, et al. Targeted delivery of antisense oligonucleotides to pancreatic $\beta$-cells. Sci Adv . 2018;4(10):eaat3386.

102. Qian ZM, Li H, Sun H, Ho K. Targeted drug delivery via the transferrin receptor-mediated endocytosis pathway. Pharmacol Rev. 2002;54(4):561-587.

103. Dautry-Varsat A, Ciechanover A, Lodish HF. pH and the recycling of transferrin during receptormediated endocytosis. Proc Natl Acad Sci U S A. 1983;80(8):2258-2262.

104. Yamashiro DJ, Tycko B, Fluss SR, Maxfield FR. Segregation of transferrin to a mildly acidic $(\mathrm{pH}$ 6.5) para-Golgi compartment in the recycling pathway. Cell.1984;37(3):789-800.

105. Aisen P. Transferrin receptor 1. Int J Biochem Cell Biol. 2004;36(11):2137-2143.

106.Sugo T, et al. Development of antibody-siRNA conjugate targeted to cardiac and skeletal muscles. J Control Release. 2016;237:1-13.

107. Wagenaar TR, et al. Identification of the endosomal sorting complex required for transport-I (ESCRT-I) as an important modulator of antimiR uptake by cancer cells. Nucleic Acids Res. 2015;43(2):1204-1215.

108. Wang S, Sun H, Tanowitz M, Liang XH, Crooke ST. Annexin A2 facilitates endocytic trafficking of antisense oligonucleotides. Nucleic Acids Res. 2016;44(15):7314-7330.

109. Miller CM, Wan WB, Seth PP, Harris EN. Endosomal escape of antisense oligonucleotides internalized by stabilin receptors is regulated by Rab5C and EEA1 during endosomal maturation. Nucleic Acid Ther. 2018;28(2):86-96.

110.Stechmann B, et al. Inhibition of retrograde transport protects mice from lethal ricin challenge. Cell. 2010;141(2):231-242.

111. Ming X, et al. The small molecule Retro-1 enhances the pharmacological actions of antisense and splice switching oligonucleotides. Nucleic Acids Res. 2013;41(6):3673-3687.

112. Juliano RL, et al. Structure-activity relationships and cellular mechanism of action of small molecules that enhance the delivery of oligonucleotides. Nucleic Acids Res. 2018;46(4):1601-1613.

113. Butler M, et al. Phosphorothioate oligodeoxynucleotides distribute similarly in class A scavenger receptor knockout and wild-type mice. JPharmacol Exp Ther. 2000;292(2):489-496.

114. Wang S, et al. Cellular uptake mediated by epidermal growth factor receptor facilitates the intracellular activity of phosphorothioate-modified antisense oligonucleotides. Nucleic Acids Res. 2018;46(7):3579-3594.

115. Orellana EA, Tenneti S, Rangasamy L, Lyle LT, Low PS, Kasinski AL. FolamiRs: ligandtargeted, vehicle-free delivery of microRNAs for the treatment of cancer. Sci Transl Med. 2017;9(401):eaam9327.

116. Zhou W, et al. Efficient intracellular delivery of oligonucleotides formulated in folate receptor-targeted lipid vesicles. Bioconjug Chem. 2002;13(6):1220-1225.

117. St-Pierre G, et al. Synthesis and biological evaluation of sialyl-oligonucleotide conjugates targeting leukocyte $B$ trans-membranal receptor $\mathrm{CD} 22$ as delivery agents for nucleic acid drugs. Bioorg Med Chem. 2016;24(11):2397-2409.

118. Satake N, et al. Novel targeted therapy for precursor B cell acute lymphoblastic leukemia: antiCD22 antibody-MXD3 antisense oligonucleotide conjugate. Mol Med. 2016;22:632-642. 